Małgorzata Słota*

\title{
Decomposition Techniques for Full-waveform Airborne Laser Scanning Data**
}

\section{Introduction}

Nowadays, since they provide fast and efficient data acquisition, airborne laser scanning techniques are widely used in many applications. Currently, besides the traditional, discrete LiDAR systems, there are more advanced systems on the market - so-called full-waveform systems.

Small-footprint full-waveform laser scanners have been available since 2004, when Riegl presented the world's first commercial digitizing and full-waveform processing airborne laser scanner, LMS-Q560 [14]. The main feature that distinguishes full-waveform from a conventional discrete system is the possibility to register both emitted and backscattered laser signal at high sampling frequencies of around $1 \mathrm{GHz}$. Therefore restoring a full profile of the reflected energy is achievable (Fig. 1).

In traditional techniques, the echo detection process is done automatically online, most often by simple thresholding methods - the manufactures however do not provide the end user with information about the used algorithm, so the precision of the range estimation is practically unknown. An example presenting simple thresholding results is shown in Figure 2.

It should be noted that the obtained point cloud does not perfectly match the scene. Secondly, neither weak nor overlapping echoes were detected. Moreover, by comparing the point cloud with the entire energy profile, it can be observed that full-waveform data holds information about the properties of reflecting objects in addition to geometry information (i.e. amplitude of recorded energy implies surface reflectivity and echo width may indicate target's roughness).

* AGH University of Science and Technology, Faculty of Mining Surveying and Environmental Engineering, Department of Geoinformation, Photogrammetry and Remote Sensing of Environment, Krakow, Poland

** This study has been carried out within the scope of research project AGH no. 11.11.150.94 
To sum up, by processing full-waveform data the end user has the ability to control the echo detection method, which can significantly improve point cloud accuracy. On the other hand, by waveform shape parameters determination, the point cloud could be enriched by additional attributes characterizing the type of target surface.
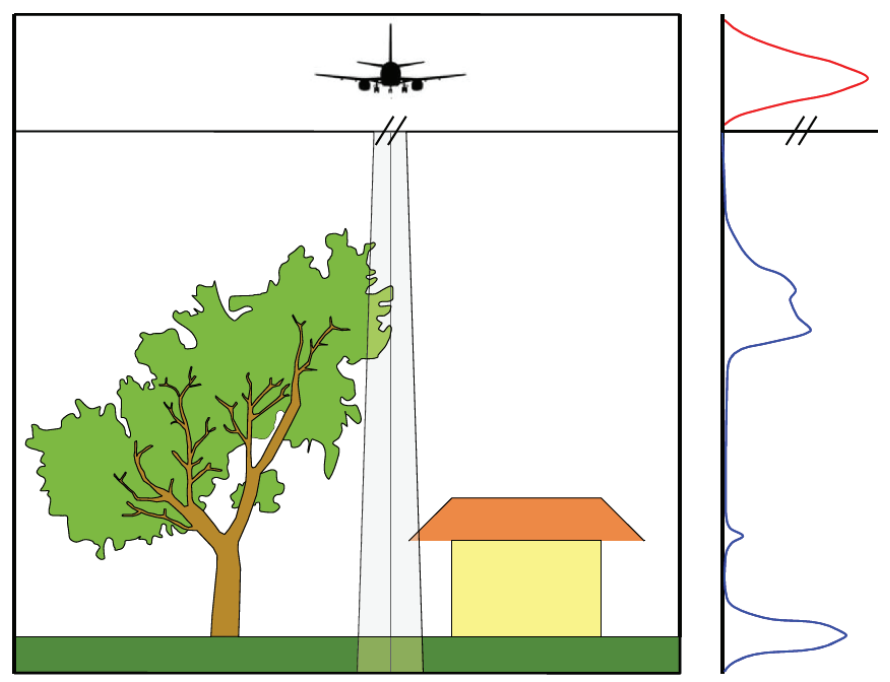

Emitted Pulse

Pulse Backscattered from Tree

Pulse Reflected

from Roof

Terrain Pulse

Fig. 1. Principles of full-waveform data acquisition
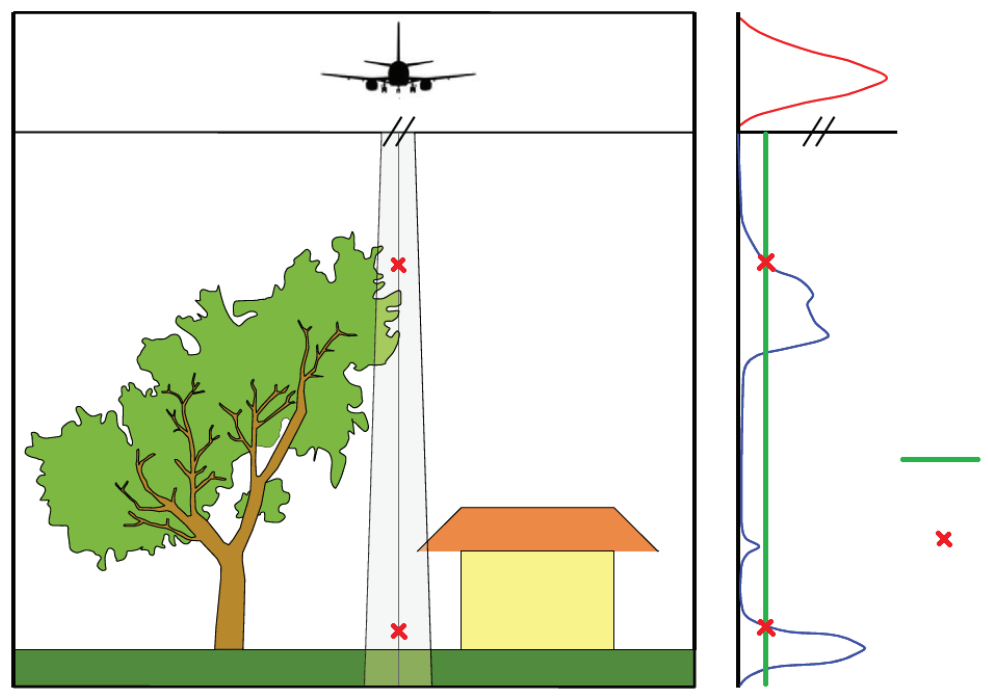

Threshold

Detected Echo

Fig. 2. Principles of simple thresholding method 


\section{Processing Methods}

The basic description of the measurement process is given by the radar equation [18]:

where:

$$
P_{r}=\frac{P_{t} \cdot D_{r}^{4}}{4 \cdot \pi \cdot R^{4} \cdot \beta_{t}^{2}} \cdot \eta_{\mathrm{sys}} \cdot \eta_{\mathrm{atm}} \cdot \sigma
$$

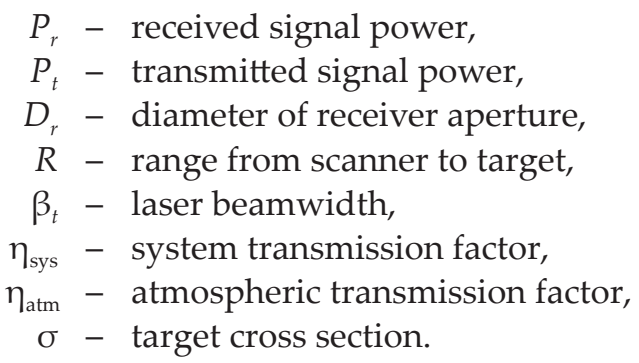

An undoubted advantage of full-waveform data processing is the potential ability to extract additional points, not detected by the online system. However, critical processing situations may occur in areas with complex targets (e.g. vegetation), where data includes many overlapping echoes or when registered echoes are weak and hardly detectable from the background noise as well as in the case of a strongly distorted signal [6].

The following sections show the three most popular methods of full-waveform data processing: the simple peak detection method, the signal decomposition approach and correlation techniques. Obviously, the point cloud extraction from registered energy profiles is the main goal of full-waveform data processing, and each of the approaches presented below achieves that goal. However, these methods differ in accuracy of range estimation and processing speed. Furthermore, by using these techniques some other, additional benefits can be obtained.

Simple peak detection methods could result in not very accurate point cloud determination, but definitely are the leaders in terms of computation speed. Signal decomposition by fitting parametric function into data is one of the most timeconsuming full-waveform data processing techniques. This method requires initial parameter estimation as well as an optimization step, nevertheless, it allows us to retrieve some additional waveform features. Using correlation techniques permits us to reduce the impact of initial values estimation. Good range determination can be achieved, but some important information of the waveform is omitted [21].

\subsection{Simple Peak Detection Methods}

The most basic technique for pulse detection is a simple thresholding method, relying on triggering a pulse whenever the rising edge of the signal exceeds a specified threshold value [18]. While defining this threshold value, the noise level as well as 
amplitude and width of the signal should be taken into account, otherwise using this method could result in large position errors (Fig. 2). In the literature, another description of peak thresholding can be found as well. In [16], Toth et al. use peak thresholding term for the method based on the assumption that the values around the peak are always lower than the peak value in a specified interval. Other quite popular methods are centre of gravity, zero crossing of the first derivative or constant fraction detection. These techniques have been investigated in [5, 18]. Centre of gravity, also called average time value detection, is a method where the temporal centre of gravity of the pulse waveform is determined [5]. The zero crossing method is a typical mathematical approach for finding local maxima in the signal. It relies on checking the sign change of the first derivative. This method is very sensitive to noise present in the signal, which is the reason why noise filtering should be applied prior to any processing. To limit the degradation of the pulse detection process performance, the amount of filtering should consider the actual signal noise level [16]. With the constant fraction detection (CFD) the constant fraction signal is computed by adding a waveform inverted and delayed by fixed time interval to the original signal. Afterwards, the zero crossing point of the calculated signal, representing peak location, is determined.

The most important advantage of these simple detectors is the processing speed. However, the quality of results strongly depends on the selected method and signal shape. That is why these simple detectors are neither appropriate for complex shape signals processing nor for tasks where geometry has to be provided at the highest accuracy. Nevertheless, these methods work well in pre-processing for the determination of initial values.

\subsection{Signal Decomposition Using Different Types of Base Functions}

The signal registered by a full-waveform system is a time-dependent function of the energy reflected at different objects within the laser cone. In order to find individual targets characteristics, signal decomposition can be performed. This decomposition process allows us for precise range identification, while the modelling of each echo with suitable analytical functions permits us to retrieve the waveform shape, which can provide additional features for segmentation or classification purposes [10]. In general, decomposition is a parametric approach to estimate a mathematical model [2]. It aims at presenting a return signal as a sum of components corresponding with targets within the laser beam travel path:

$$
y=f(x)=\sum_{j=1}^{n} f_{j}(x)
$$

where:

$n$ - number of components,

$f_{j}-$ analytical function. 
Current practice for signal modelling uses the Gaussian function, generalized Gaussian, Lognormal, Weibull, Nakagami or Burr functions.

\section{Waveform decomposition by Gaussian function}

Since most of the emitted laser pulses are nearly Gaussian in their shape, the reflected signal for simple surfaces is also nearly Gaussian. For data over surfaces with multiple scatterers along the laser line-of-sight the return signal can be modelled as a mixture of Gaussian components [12]. In fact, the best-known and most frequently used parametric function for full-waveform airborne laser scanning data decomposition is the Gaussian function:

$$
f_{G, j}(x)=I_{j} \cdot \exp \left(-\frac{\left(x-\mu_{j}\right)^{2}}{2 \sigma_{j}^{2}}\right)
$$

where:

$I_{j}$ - echo amplitude,

$\mu_{j}-$ echo position (shift),

$\sigma_{j}-$ standard deviation (echo width).

The Gaussian distribution is presented in the Figure 3.

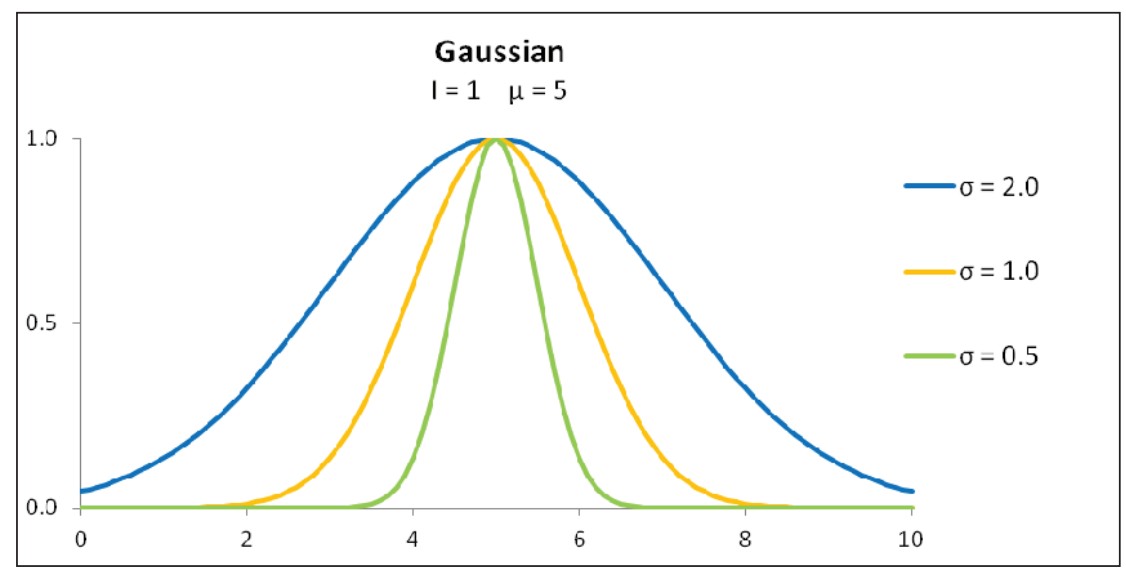

Fig. 3. The Gaussian distribution

The Gaussian function has three parameters and can be used to model symmetric echoes. Gaussian decomposition is sufficient for most applications, especially for large-footprint LiDAR data, and although modelling results strongly depends on initial parameters, it has been shown that $98 \%$ of the full-waveform data could be successfully modelled using Gaussian pulses [17]. 


\section{Generalized Gaussian model}

Another commonly used tool for data modelling is an extension of the Gaussian model (Fig. 4), namely the generalized Gaussian function (GG), defined as:

$$
f_{G G, j}(x)=I_{j} \cdot \exp \left(-\frac{\left|x-\mu_{j}\right|^{\alpha_{j}^{2}}}{2 \sigma_{j}^{2}}\right)
$$

where $\alpha_{j}$ - flatness parameter.

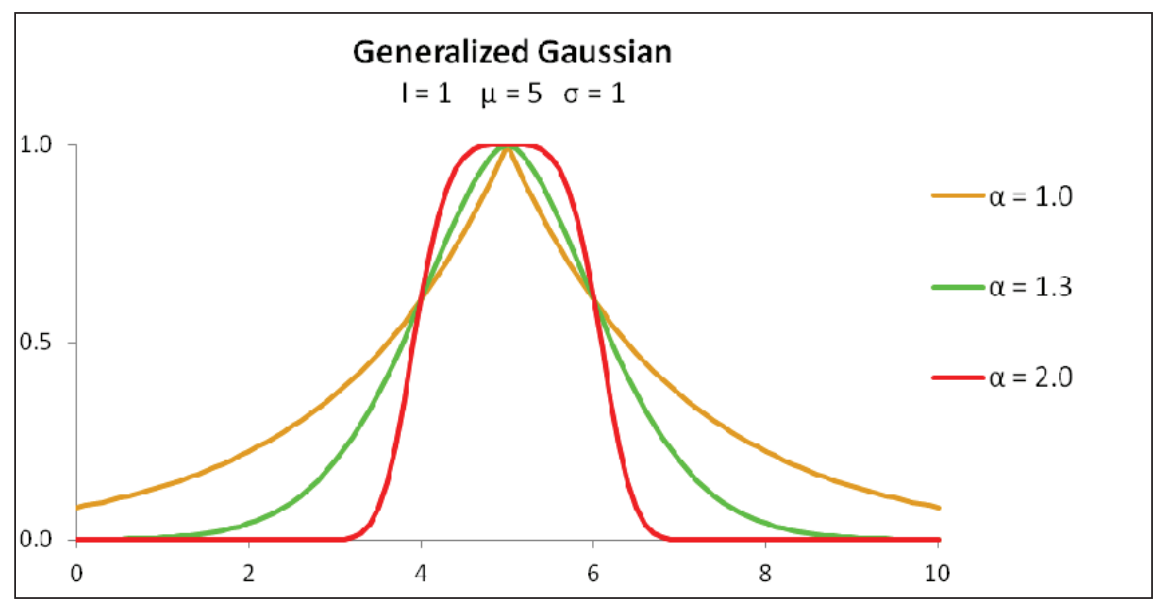

Fig. 4. The generalized Gaussian (GG) distribution

The generalized Gaussian model, like the Gaussian one, only permits to handle symmetric echoes. However, the additional shape parameter a is responsible for controlling the waveform flatness, which is very beneficial for modelling the distorted signal occurring over forested areas and over some building roofs [2]. For $1 \leq \alpha<\sqrt{2}$ the generalized Gaussian shape is peaked in comparison to the Gaussian, $\alpha=\sqrt{2}$ results in the Gaussian function, whereas for $\alpha>\sqrt{2}$ the curve is flattened.

More about the results on generalized Gaussian fitting can be found in $[2,8,10]$.

\section{Decomposition by the lognormal function}

Besides symmetric echoes full-waveform data can include slightly asymmetric pulse responses. Consequently, waveform approximation by the sum of Gaussians or generalized Gaussians could lead to an inaccurate representation. However, these backscatterers can be modelled by an asymmetric base function like the lognormal (Fig. 5). 
The lognormal function is described by the following equation:

$$
f_{L, j}(x)=I_{j} \cdot \exp \left(-\frac{\left(\ln \left(x-\mu_{j}\right)-s_{j}\right)^{2}}{2 \sigma_{j}^{2}}\right)
$$

where:

$\mu_{j}-$ location parameter,

$\sigma_{j}-$ standard deviation (shape),

$s_{j}-$ scale parameter.

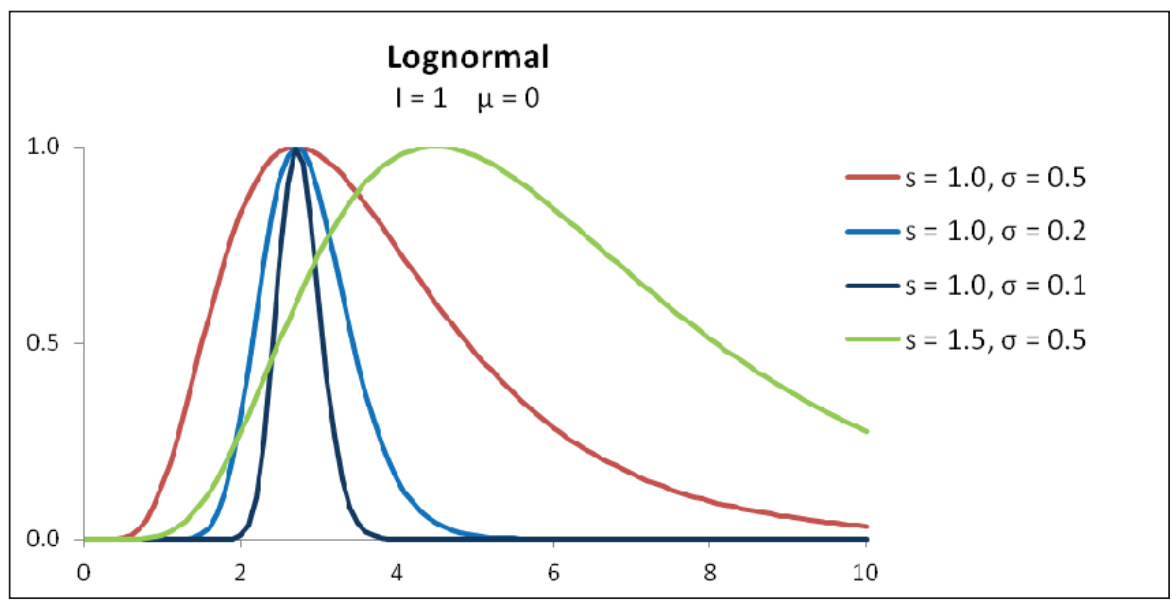

Fig. 5. Lognormal function family

It was proven in [2] that modelling raw signals only with Lognormal functions is not suitable for the whole, heterogeneous survey area, but can improve the waveform fitting locally, where asymmetric echoes are observed. Such cases occur especially on streets and some building roofs [2].

\section{Waveform decomposition by Weibull modelling}

Another function family allowing us to model asymmetric backscatter pulse reflections is the Weibull family [10] (Fig. 6), defined by:

$$
f_{W, j}(x)=I_{j} \cdot \frac{k_{j}}{\lambda_{j}} \cdot\left(\frac{x-\mu_{j}}{\lambda_{j}}\right)^{k_{j}-1} \cdot \exp \left(-\frac{x-\mu_{j}}{\lambda_{j}}\right)^{k_{j}}
$$

where:

$k_{j}-$ shape parameter,

$\lambda_{j}-$ scale parameter. 


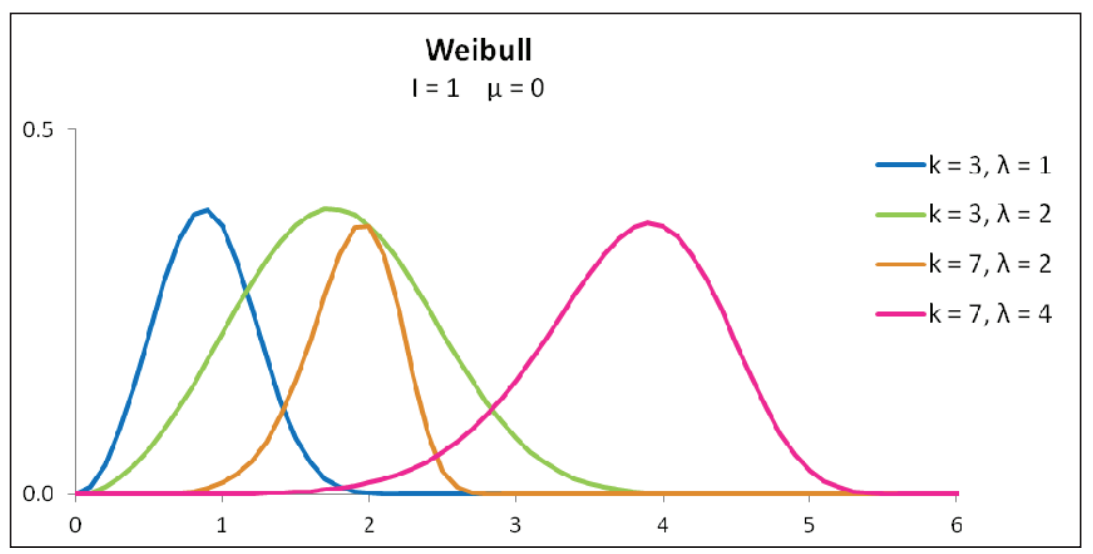

Fig. 6. Weibull distribution

\section{The Nakagami model}

The Nakagami model was introduced by Nakagami in the early 1940's [15] (Fig. 7). It is a probability distribution related to the gamma distribution, given by:

$$
f_{N, j}(x)=I_{j} \cdot \frac{2 \xi_{j}^{\xi_{j}}}{\omega_{j} \Gamma\left(\xi_{j}\right)} \cdot\left(\frac{x-\mu_{j}}{\omega_{j}}\right)^{2 \xi_{j}-1} \cdot \exp \left(-\xi_{j}\left(\frac{x-\mu_{j}}{\omega_{j}}\right)^{2}\right)
$$

where:

$\xi_{j}-$ scale parameter,

$\omega_{j}-$ skewness parameter,

$\Gamma\left(\xi_{j}\right)$ - the Gamma function.

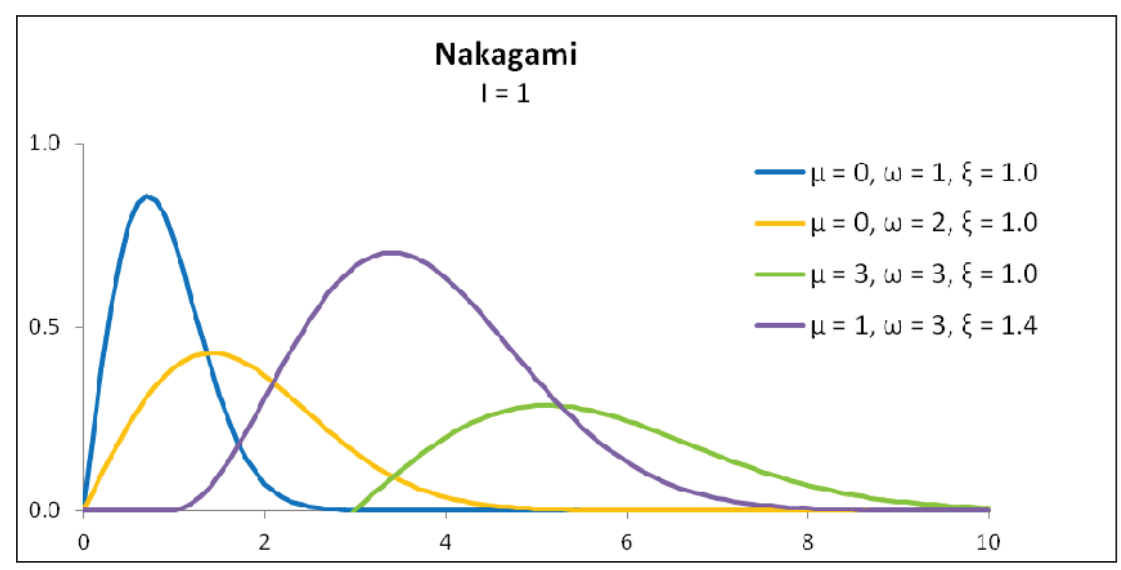

Fig. 7. Nakagami distribution 
The Nakagami model for full-waveform data processing has been introduced in order to model soft right-skewed and left-skewed behaviours [8]. More about the results of the Nakagami fitting can be found in [10].

\section{Burr function}

The Burr distribution (Fig. 8) was firstly introduced in 1942 by Irving W. Burr as a two-parameter function family. An additional scale parameter was presented in 1980 by Tadikamalla. It is a very flexible function family that can express a wide range of waveform shapes [19]. The Burr function is given by the equation:

$$
f_{B, j}(x)=I_{j} \cdot \frac{b_{j} c_{j}}{a_{j}} \cdot\left(\frac{x-\mu_{j}}{a_{j}}\right)^{-b_{j}-1} \cdot\left(1+\left(\frac{x-\mu_{j}}{a_{j}}\right)^{-b_{j}}\right)^{-c_{j}-1}
$$

where:

$$
\begin{aligned}
& a_{j}-\text { scale parameter }(>0), \\
& b_{j}-\text { shape parameter }(>0), \\
& c_{j}-\text { shape parameter }(>0) .
\end{aligned}
$$

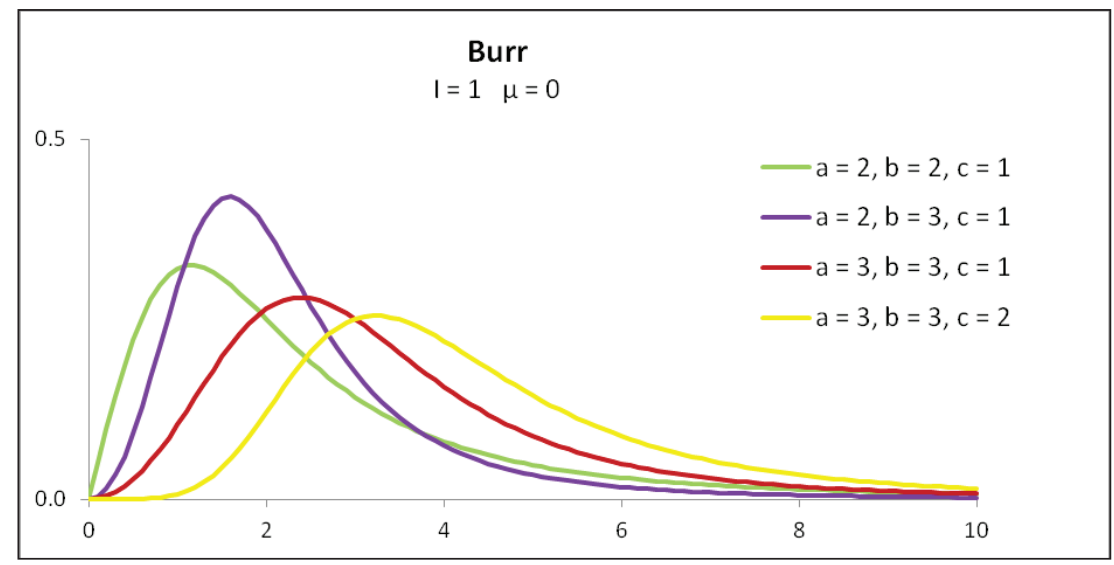

Fig. 8. The Burr function family

Different parameter values can cover a broad set of skewness, which is why the Burr function is perfect for model asymmetric waveforms, especially right-skewed.

Typically, the decomposition of full-waveform signal, regardless of the chosen base function, includes two main steps. Firstly, the number of reflections is estimated, along with information about echo localization, echo amplitude or other parameters. Initial values can be obtained by one of the simple peak detection methods, however, these results are very sensitive to noise present in the signal. Therefore, denoising or signal smoothing is commonly used as one of the pre-processing stages. 
A few examples of initial valu estimation are presented below:

- In [17] Wagner et al. use the centre of gravity and zero-crossing of the first derivative methods for initial values extraction.

- The deterministic nonparametric "bump-hunting" procedure, providing the number, amplitudes, and positions of the suspected returns, is mentioned in [3].

- In [20] the initial values for amplitude and timing point are calculated after conducting a specific search procedure for the detection of visible and overlapping peaks. Firstly, the local maxima above the defined threshold separating the signal from background noise are found. Then, the algorithm looks for inflexion points on both sides of the primary visible peaks. An initial width value for each component is set to the width of the transmitted pulse.

- In [13] initial estimates are derived by combining the peak thresholding method (called the maximum detection method) with zero crossing method.

- The zero crossing of the first derivative technique is used as well in [2]. This peak detection method is applied to the thresholded version of the waveform. In addition, the proposed algorithm takes into account a minimal number of samples separating two detectable peaks (spatial resolution of the system).

- In [11] the number of peaks is determined by inspecting the second derivative of a cubic smoothing spline fitted to the waveform data.

In the second step, one of the optimization methods is carried out to fit the data with single modelling function to obtain the final estimates of echo parameters. Currently, there are three typically used optimization approaches:

1) the non-linear least-square method using the Levenberg-Marquardt algorithm,

2) the maximum likelihood estimate with the expectation-maximization algorithm,

3) the stochastic approach based on the reversible jump Monte Carlo Markov chain model [9].

\subsection{Signal Correlation Techniques}

Unfortunately, the shapes of the backscattered overlapping echoes usually differ from the assumed model [20]. Additionally, modelling is much more complicated when the signal is distorted by a high level of noise or when the data include artificial echoes caused by the so-called "ringing effect". This effect is due to the bandwidth limitation of the receiver electronics and can be observed as a smaller peak right after the main peak [13] (Fig. 9).

To overcome issues mentioned above, another approach of full-waveform data processing is proposed. These techniques are based on the transmitted and received waveform comparison. Firstly, a proper correlation function is computed. Then, the local maxima (minima), representing echoes of the emitted laser pulse, have to be 
detected. This can be performed by using one of the simple peak detection methods, or, for example, a method based on a few user defined criteria with parabola fitting, as presented in [13].

a)

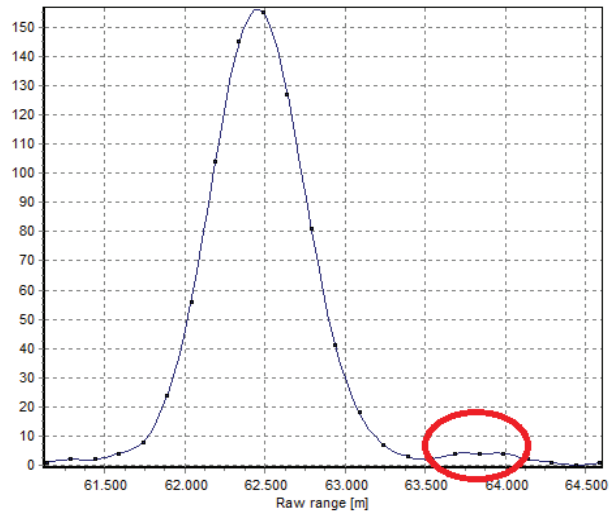

b)

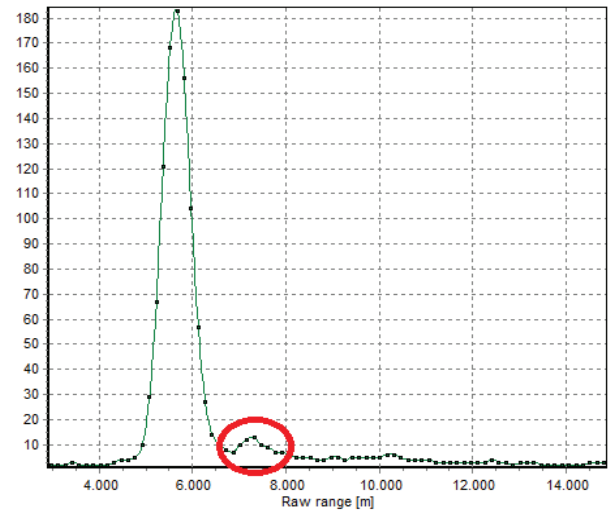

Fig. 9. "Ringing effect" in Riegl LMS-Q680i data:

a) emitted laser pulse; b) backscatter signal

In the first approach, the normalized cross-correlation $R_{\text {aver }}$ between the emitted pulse waveform $s(t)$ and the received waveform $r(t)$ of the backscattered echo is calculated [1]:

$$
R_{\text {aver }}(\tau)=\frac{\int_{t=-\infty}^{\infty} s(t) \cdot r(t+\tau) d t}{\sqrt{\int_{t=-\infty}^{\infty} s^{2}(t) d t \cdot \int_{t=-\infty}^{\infty} r^{2}(t) d t}}
$$

The second method analytically comparable with the direct correlation is called the averaged square difference function (ASDF). The ASDF is a time delay estimation technique based on the correlation of the reference signal $s(t)$ and the measured signal $r(t)$ [4]. In full-waveform data processing $s(t)$ and $r(t)$ are equidistanced time series with sampling interval T. The ASDF response value $R_{A S D F}(\tau)$ between $s(t)$ and the shifted waveform $r(t+\tau)$ is defined as follows [4]:

$$
R_{A S D F}(\tau)=\frac{1}{N} \sum_{k=1}^{N}(s(k T)-r(k T+\tau))^{2}
$$

where $\tau$ - time shift, $\tau=-N T,(-N+1) T, \ldots, N T$ is an integral multiple of $T$.

The ASDF method is equivalent to direct correlation (DC) if normalized in the interval $[0,1][13]$. The minimum of the ASDF function corresponds to the maximum of correlation coefficient. 
For both techniques, the calculated distance is improved, compared to the peak detection analysis of the received signal [7]. Moreover, the noise and the "ringing effect" influence can be significantly reduced.

\section{Summary}

Full-waveform airborne laser scanning becomes more and more popular. While full-waveform digitization technology is already well developed, the data processing methods are still in the research stage.

For rough but fast point cloud extraction from full-waveform profiles, one of the simple echo detection methods, such as zero crossing of the first derivative or peak thresholding, can be used. If high accuracy of the point cloud is required and the detection of a maximum number of echoes is essential, signal decomposition or methods based on signal correlation should be applied. Both these techniques are more time consuming, but it was shown that they can give much better results. When full-waveform LiDAR data is used for classification purposes, extracting additional features during the signal decomposition process is recommended. The calculated set of full-waveform features depends on the type of function used for signal modelling. The most popular signal decomposition approach consists in presenting a waveform as a sum of Gaussian components, and allows us to retrieve the echo position, amplitude and width. Moreover, the full-waveform data can be modelled with generalized Gaussian, Lognormal, Weibull, Nakagami or Burr functions. The correlation techniques work well for data affected by high noise level or the "ringing effect", however, some important waveform features are not included.

Extensive research on the potential of the LiDAR full-waveform data will certainly continue in the coming years. The use of such data for forestry applications has been fairly well studied over the last ten years. The developed algorithms for creating detailed descriptions of vegetated structures will be improved in the future as well as methods for modelling single trees. The potential of full-waveform data use in the urban areas has not yet been fully exploited. Therefore, the full-waveform processing techniques development will probably be focused on this issue, especially on the improvement in the separation between vegetation and buildings, and precise building edge detection methods.

\section{References}

[1] Bretar F., Chauve A., Mallet C., Jutzi B.: Managing full waveform LIDAR data: A challenging task for the forthcoming years. The Internal Archives of the Photogrammetry, Remote Sensing and Spatial Information Sciences, vol. XXXVII-B1, 2008, pp. 415-420. 
[2] Chauve A., Mallet C., Bretar F., Durrieu S., Deseilligny M.P., Puech W.: Processing full-waveform lidar data: modelling raw signals. The International Archives of Photogrammetry, Remote Sensing and Spatial Information Sciences, vol. XXXVI-3/W52, 2007, pp.102-107.

[3] Hernandez-Marin S., Wallace A.M., Gibson G.J.: Bayesian analysis of lidar signals with multiple returns. Pattern Analysis and Machine Intelligence, IEEE Transactions on, vol. 29(12), 2007, pp. 2170-2180.

[4] Jacovitti G., Scarano G.: Discrete time techniques for time delay estimation. Signal Processing, IEEE Transactions on, vol. 41(2), 1993, pp. 525-533.

[5] Jutzi B., Stilla U.: Laser pulse analysis for reconstruction and classification of urban objects. The International Archives of the Photogrammetry, Remote Sensing and Spatial Information Sciences, vol. XXXIV-3/W8, 2003, pp. 151-156.

[6] Jutzi B., Stilla U.: Range determination with waveform recording laser systems using a Wiener Filter. ISPRS Journal of Photogrammetry and Remote Sensing, vol. 61(2), 2006, pp. 95-107.

[7] Kirchhof M., Jutzi B., Stilla U.: Iterative processing of laser scanning data by full waveform analysis. ISPRS Journal of Photogrammetry and Remote Sensing, vol. 63(1), 2008, pp. 99-114.

[8] Mallet C., Bretar F., Roux M., Soergel U., Heipke C.: Relevance assessment of full-waveform lidar data for urban area classification. ISPRS Journal of Photogrammetry and Remote Sensing, vol. 66(6), supplement, 2011, pp. S71-S84.

[9] Mallet C., Bretar F.: Full-waveform topographic lidar: State-of-the-art. ISPRS Journal of Photogrammetry and Remote Sensing, vol. 64(1), 2009, pp. 1-16.

[10] Mallet C., Lafarge F., Bretar F., Soergel U., Heipke C.: Lidar waveform modeling using a marked point process. Image Processing (ICIP), IEEE, $16^{\text {th }}$ IEEE International Conference, 2009, pp. 1713-1716.

[11] Molnar B., Laky S., Toth C.: Using Full Waveform Data in Urban Areas. The International Archives of the Photogrammetry Remote Sensing and Spatial Information Sciences, vol. XXXVIII-3/W22, 2011, pp. 203-208.

[12] Neuenschwander A., Magruder L., Gutierrez R.: Signal Processing Techniques for Feature Extraction and Classification using Small-Footprint FullWaveform Airborne LIDAR. Geoscience and Remote Sensing Symposium, IEEE International , vol. 3, 2008, pp. 676-679.

[13] Roncat A., Wagner W., Melzer T., Ullrich A.: Echo detection and localization in full-waveform airborne laser scanner data using the averaged square difference function estimator. The Photogrammetric Journal of Finland, vol. 21(1), 2008, pp. 62-75.

[14] Słota M.: Prospects for improving the buildings edge detection algorithms based on full-waveform LIDAR information compared with the traditional multiecho laser scanner data. $4^{\text {th }}$ Doctoral Seminar on Geodesy and Cartograpy, Olsztyn, Poland, Wydawnictwo UWM, 2011, pp. 99-105. 
[15] Stuber G.L.: Pronciples of Mobile Comunication. Springer, 2001.

[16] Toth Ch.K., Zaletnyik P., Laky S., Grejner-Brzezinska D.A.: Peak detection from full-waveform LiDAR data. International LiDAR Mapping Forum, New Orleans, Louisiana, February 7-9, 2011.

[17] Wagner W., Ullrich A., Ducic V., Melzer T., Studnicka N.: Gaussian decomposition and calibration of a novel small-footprint full-waveform digitising airborne laser scanner. ISPRS Journal of Photogrammetry and Remote Sensing, vol. 60(2), 2006, pp. 100-112.

[18] Wagner W., Ullrich A., Melzer T., Briese C., Kraus K.: From single-pulse to full-waveform airborne laser scanners: potential and practical challenges. The International Archives of Photogrammetry and Remote Sensing, vol. XXXV-B3, 2004, pp. 201-206.

[19] www.mathworks.com.

[20] YuChing L., Mills J., Smith-Voysey S., Hill R., Rosette J., Suárez J.: Detection of weak and overlapping pulses from waveform airborne laser scanning data. SilviLaser 2008, $8^{\text {th }}$ International Conference on LiDAR Applications in forest assessment and inventory, Heriot-Watt University, Edinburgh, UK, 17-19 September, 2008, pp. 478-487.

[21] Zhu J., Zhanga Z., Hu X., Lia Z.: Analysis and application of LiDAR waveform data using a progressive waveform decomposition method. The International Archives of the Photogrammetry, Remote Sensing and Spatial Information Sciences, vol. XXXVIII-5/W12, 2011, pp. 31-36. 